Proyecciones

Vol. $1.3 N^{\circ} 2$, pp. 85-97 December 1994

Iniversidad Católica del Norte

Antofagasta - Chile

\title{
THE WEAK SOLUTIONS AND REPRODUCTIVE PROPERTY FOR A SYSTEM OF EVOLUTION EQUATIONS OF MAGNETOHYDRODYNAMIC TYPE
}

\author{
MARKO A. ROJAS-MEDAR and JOSE LUIZ BOLDRINI \\ IMECC - UNICAMP, Campinas, SP, Brazil
}

\begin{abstract}
By using the Galerkin method, we prove the existence of weak solutions for a system of equations of magnetohydrodynamic type. In the two-dimensional case, we prove that the weak solution is unique. We also prove the reproductive property and comment about the regularity of periodic solutions.
\end{abstract}




\section{Introduction}

In several situations the motion of incompressible electrical fluids can be modelled by the so called equations of magnetohydrodynamics, which correspond to the Navier-Stokes equations coupled with the Maxwell's equations. In the case where there is free motion of heavy ions, not directly due to the electrical field (see Schlüter [11] and Pikelner [9]), these equations c an be reduced to the following form:

$$
\left\{\begin{array}{l}
\frac{\partial u}{\partial t}-\frac{\eta}{\rho_{m}} \Delta u+u . \nabla u-\frac{\mu}{\rho_{m}} h . \nabla h=f-\frac{1}{\rho_{m}} \nabla\left(p^{*}+\frac{\mu}{2} h^{2}\right), \\
\frac{\partial h}{\partial t}-\frac{1}{\mu \sigma} \Delta h+u . \nabla h-h . \nabla u=-\operatorname{grad} w, \\
\operatorname{div} u=0, \\
\operatorname{div} h=0 .
\end{array}\right.
$$

Here, $u$ and $h$ are respectively the unknown velocity and magnetic fields; $p^{*}$ is the unknown hydrostatic pressure; $w$ is an unknown function related to the motion of heavy ions (in such way that the density of electric current, $j_{0}$, generated by this motion satisfies the relation $\left.\operatorname{rot} j_{0}=-\sigma \nabla w\right) ; \rho_{m}$ is the density of mass of the fluid (assumed to be a positive constant); $\mu>0$ is the constant magnetic permeability of the medium; $\sigma>0$ is the constant electric conductivity; $\eta>0$ is the constant viscosity of the fluid; $f$ is a given external force field. These equations are supposed to be valid in a region $\Omega \subset \mathbb{R}^{n}, n \geq 2$, and an interval of time $[0, T)$.

To (1.1) we append the following boundary and initial conditions

$$
\begin{aligned}
& \left.u\right|_{\partial \Omega}=0 \quad \text { and }\left.\quad h\right|_{\partial \Omega}=0, \\
& u(0)=u_{0} \quad \text { and } \quad h(0)=h_{0},
\end{aligned}
$$

where for simplicity we have considered homogeneous boundary conditions; $u_{0}, h_{0}$ are given functions.

The stationary problem corresponding to (1.1) was considered by Chizhonkov [4], while the question of (local) existence of solution of the evolution problem (1.1) was analyzed by Lassner [7] by making the use of semigroup techniques similar to ones in Fujita and Kato [5] (he also studied the asymptotic behavior of the solution as $t \rightarrow 0^{+}$).

The more constructive spectral Galerkin method was used by Boldrini and Rojas-Medar [3] to obtain local in time strong solutions and to study conditions for regularity for $t>0$ (see [2]) and by Rojas-Medar and Boldrini [10] to obtain global strong solutions. Here the word spectral is used in the sense that the eigenfunctions of the associated Stokes operator are used as a basis of approximation. The authors in [2], [3], [7], [10] work only for $n=2$ or 3 . 
In this paper we will consider the problem of existence and uniqueness of weak solutions of as well as the question about the reproductive property for (1.1)-(1.3). The argument are based on the construction of approximate solutions by the Galerkin method and, by taking the limit with the use of a priori estimates and a compactness theorem (cf. [8]), we show that there are weak solutions. We also prove that the two-dimensional problems is uniquely solvable.

Let $\{u, h\}$ be a weak solution of (1.1) and (1.2) (the exact definition will be given later on). Given $T>0$, if there are weak solutions $u$ and $h$ satisfying the following condition:

$$
u(x, 0)=u(x, T) \quad, \quad h(x, 0)=h(x, T),
$$

then we say that the system has the reproductive property (see [6] for the case of the Navier-Stokes equations). We observe that the above property is a generalization of the notion of periodicity. We will show that (1.1)-(1.3) has always a weak solution with the reproductive property.

Finally, the paper is organized as follows: in Section 2 we state the basic assumptions and results to be used later in the paper; we also rewrite (1.1) and (1.2) in a more suitable weak form; we describe the approximation method and state the results of the paper (Theorems 2.1, 2.2, and 2.3). Each one of the following sections will be devoted to their proofs.

\section{Preliminaries and Results}

Let $\Omega \subseteq \mathbb{R}^{n}, n \geq 2$, be a bounded domain with smooth boundary $\partial \Omega$. We denote by $L^{p}(\Omega)$ the usual Lebesgue spaces and by $\|\cdot\|_{L^{p}}$ the $L^{p}$-norm on $\Omega$, in the case $p=2$, we simply denote the $L^{2}$-norm by $|\cdot|$ and the inner product by $(\cdot, \cdot)$. With the same symbols we denote the spaces of $n$-dimensional vector functions. If $B$ is a Banach-space, we denote by $L^{q}(0, T ; B)$ the Banach space of the $B$-valued functions defined in the interval $(0, T)$ that are $L^{q}$-integrable in the sense of Bochner. The Sobolev spaces, $H^{s}(\Omega), \quad H_{0}^{s}(\Omega)(s \in \mathbb{R})$ are defined as usual, we denote their norm by $\|\cdot\|_{s}$ and by $(,)_{H^{s}}$ their inner product. We also will use the following solenoidal function spaces

$$
\begin{aligned}
& C_{0, \sigma}^{\infty}(\Omega)=\left\{v \in\left(C_{0}^{\infty}(\Omega)\right)^{n} / \operatorname{div} v=0 \text { in } \Omega\right\} \\
& H=\text { the completion of } C_{0, \sigma}^{\infty}(\Omega) \text { under the } L^{2} \text {-norm. } \\
& V_{s}=\text { the completion of } C_{0, \sigma}^{\infty}(\Omega) \text { under the } H^{s} \text {-norm. }
\end{aligned}
$$

In the special case where $s=1$, we denote $V_{1}$ simply by $V$.

The norm and inner product in $H$ and $V_{s}$ are:

$$
(f, g)=\sum_{i=1}^{n} \int_{\Omega} f_{i} g_{i} d x \quad, \quad|f|=(f, f)^{1 / 2}
$$


and

$$
(u, v)_{s}=\sum_{i=1}^{n}\left(u_{i}, v_{i}\right)_{H^{s}} \quad, \quad\|u\|_{s}=(u, u)_{s}^{1 / 2} .
$$

If $X$ is a Banach space, $X^{*}$ will denote the topological dual of $X$.

We observe that $V$ is characterized by

$$
V=\left\{v \in\left(H_{0}^{1}(\Omega)\right)^{n} \mid \operatorname{div} v=0 \text { in } \Omega\right\},
$$

and consequently the $H^{1}$-norm and $H_{0}^{1}$-norm are equivalent for $u \in V$. We denote $\|u\|=|\nabla u|$, that is, $\|u\|=\left(\int_{\Omega}|\nabla u|^{2}\right)^{1 / 2}$.

On the other hand, let us set

$$
\begin{aligned}
& a(v, w)=\sum_{i, j=1}^{n} \int_{\Omega} \frac{\partial v_{j}}{\partial x_{i}} \frac{\partial w_{j}}{\partial x_{i}} d x, \\
& b(u, v, w)=\sum_{i, j=1}^{n} \int_{\Omega} u_{j} \frac{\partial v_{i}}{\partial x_{j}} w_{i} d x,
\end{aligned}
$$

which we define for all vector-valued functions $u, v$ and $w$, for which the integrals are well-defined.

We can now define a notion of weak solution for (1.1)

Definition. Let $u_{0}, h_{0} \in H$; we will say that a couple of functions $(u, h)$ defined on $\Omega \times(0, T)$ is a weak solution of $(1.1)-(1.3)$ if and only if $u, h \in$ $L^{2}(0, T ; V) \cap L^{\infty}(0, T ; H)$ and

$$
\left\{\begin{array}{l}
\alpha\left(u_{t}, \phi\right)+\nu a(u, \phi)+\alpha b(u, u, \phi)-b(h, h, \phi)=(\alpha f, \phi) \\
\left(h_{t}, \psi\right)+\gamma a(h, \psi)+b(u, h, \psi)-b(h, u, \psi)=0
\end{array}\right.
$$

for all $\left.\phi, \psi \in V \cap\left(L^{n}(\Omega)\right)\right)^{n}$. We also require that (1.3) be satisfied (as it is usual the regularity condition above is enough to guarantee that (1.3) has a meaning. By the same token, (1.4) has a meaning.

In the above formula we have denoted

$$
\alpha=\frac{\rho_{m}}{\mu} \quad, \quad \nu=\frac{\eta}{\mu}, \quad \gamma=\frac{1}{\mu \sigma} .
$$

To prove the existence of solutions of system (1.1)-(1.3) we will use the Galerkin method. We fix $s=n / 2$, and we consider the special basis $\left\{w^{i}(x)\right\}_{i=1}^{\infty}$ of $V_{n / 2}$, whose elements we choose as the solutions of the spectral problem:

$$
\left(w^{i}, v\right)_{n / 2}=\lambda_{i}\left(w^{i}, v\right) \quad \forall v \in V_{n / 2}
$$

Let $V^{k}=\operatorname{span}\left[w^{1}(x), \ldots, w^{k}(x)\right]$; we observe that $V^{k} \subseteq V_{n / 2}$. For every $k \geq 1$, we define approximations $u^{k}$ and $h^{k}$ of $u$ and $h$, respectively, by 
means of the following finite expansions:

$$
u^{k}(x, t)=\sum_{i=1}^{k} c_{i k}(t) w^{i}(x) \quad, \quad h^{k}(x, t)=\sum_{i=1}^{k} d_{i k}(t) w^{i}(x)
$$

satisfying the following equations

$$
\begin{aligned}
& \alpha\left(u_{t}^{k}, \phi\right)+\nu a\left(u^{k}, \phi\right)+\alpha b\left(u^{k}, u^{k}, \phi\right)-b\left(h^{k}, h^{k}, \phi\right)=(\alpha f, \phi) \quad \forall \phi \in V^{k} \\
& \left(h_{t}^{k}, \psi\right)+\gamma a\left(h^{k}, \psi\right)+b\left(u^{k}, h^{k}, \psi\right)-b\left(h^{k}, u^{k}, \psi\right)=0 \quad \forall \psi \in V^{k} \\
& u^{k}(0)=u_{0}^{k}, \quad h^{k}(0)=h_{0}^{k}
\end{aligned}
$$

where $u_{0}^{k} \rightarrow u_{0}$ and $h_{0}^{k} \rightarrow h_{0}$ in $H$ as $k \rightarrow \infty$.

By using these approximations, we will prove the following results.

Theorem 2.1. If $f \in L^{2}\left(0, T ; V^{*}\right), u_{0}, h_{0} \in H$, then there exist a weak solution $(u, h)$ of (1.1)-(1.3). Furthermore, $u, h \in L^{\infty}(0, T ; H)$.

Theorem 2.2. Let $n=2$. The weak solution $(u, h)$ of Theorem 2.1 is unique. Moreover, $u$ and $h$ are almost everywhere equal to a continuous function from $[0, T]$ to $H$.

Theorem 2.3. Let $f \in L^{2}\left(0, T ; V^{*}\right)$, then there exist a weak solution $(u, h)$ of (1.1)-(1.2) having the reproductive property (1.4). Furthermore, $u, h \in$ $L^{\infty}(0, T ; H)$.

Remark 1. Exactly as in [8, p. 74], it is possible to prove that the solution refered to in Theorem 2.1, after a modification in a set of zero mesure in $[0, T]$, is continuous from $[0, T] \rightarrow V_{(n-2) / 4}^{*}$ and also weakly continuous from $[0, T] \rightarrow H$.

2 . In the case where we have uniqueness of solutions for the initial value problem, as for example the two dimensional case (Theorem 2.2 ), if the external force field is regular and $T$-periodic in time, the above Theorem 2.3, furnishes a $T$-periodic weak solution for (1.1)-(1.2). In fact, it is a strong solution and actually very regular. This is so because we can prove that $u(t), h(t) \in C^{\infty}(\Omega)$ for $t>0$ and any initial data $u_{0}, h_{0} \in H$. Thus, $u_{p}(t), h_{p}(t) \in C^{\infty}(\Omega)$ for $t \in[T, 2 T]$ and by the $T$-periodicity, we conclude that $u_{p}(t)=u_{p}(t+T) \in C^{\infty}(\Omega)$ and $h_{p}(t)=h_{p}(t+T) \in C^{\infty}(\Omega)$ for $t \in[0, T]$. In particular, we must have $u_{p}(0), h_{p}(0) \in C^{\infty}(\Omega)$. 


\section{Proof of Theorem 2.1}

Concerning the existence of the solutions $\left(u^{k}, h^{k}\right)$, we observe that (2.1)(2.2) are satisfied for all $\phi, \psi \in V^{k}$ if they are satisfied for $\phi, \psi \in\left\{\omega^{1}, \ldots, \omega^{k}\right\}$. Thus, (2.1), (2.2) corresponds to a (quadratic) system of ordinary differential equations for which the usual local existence and uniqueness theorems can be applied. We conclude that for each $k \in N$ there is an maximal interval of existence $\left[0, T_{k}\right)$ where the solution $\left(u^{k}, h^{k}\right)$ exists and is unique. In the following we will show that those intervals are actually $[0, \infty)$, that is, $T_{k}=+\infty$, by showing that there is not blow up infinite time.

we obtain

In fact, by setting $\phi=u^{k}$ and $\psi=h^{k}$ in (2.1) and (2.2), respectively,

$$
\begin{aligned}
& \frac{\alpha}{2} \frac{d}{d t}\left|u^{k}\right|^{2}+\nu a\left(u^{k}, u^{k}\right)=\alpha\left(f, u^{k}\right)+b\left(h^{k}, h^{k}, u^{k}\right), \\
& \frac{1}{2} \frac{d}{d t}\left|h^{k}\right|^{2}+\gamma a\left(h^{k}, h^{k}\right)=b\left(h^{k}, u^{k}, h^{k}\right)
\end{aligned}
$$

since $b(\xi, w, w)=0$ for $w \in V^{k}$.

Adding the above equalities and observing that $b\left(h^{k}, h^{k}, u^{k}\right)+$ $b\left(h^{k}, u^{k}, b^{k}\right)=0$, we get

$$
\frac{1}{2} \frac{d}{d t}\left(\alpha\left|u^{k}\right|^{2}+\left|h^{k}\right|^{2}\right)+\nu a\left(u^{k}, u^{k}\right)+\gamma a\left(h^{k}, h^{k}\right)=\alpha\left(f, u^{k}\right) .
$$

\section{Consequently,}

$$
\frac{1}{2} \frac{d}{d t}\left(\alpha\left|u^{k}\right|^{2}+\left|h^{k}\right|^{2}\right)+\nu a\left(u^{k}, u^{k}\right)+\gamma a\left(h^{k}, h^{k}\right) \leq \frac{\nu}{2} a\left(u^{k}, u^{k}\right)+c\|f\|_{*}^{2}
$$

thanks to the Hölder and Young inequalities.

The above differential inequality implies the integral inequality,

$$
\begin{gathered}
\left.\alpha\left|u^{k}(t)\right|^{2}+\left|h^{k}(t)\right|^{2}\right)+2 \nu \int_{0}^{t}\left\|u^{k}(s)\right\|^{2} d s+2 \gamma \int_{0}^{t}\left\|h^{k}(s)\right\|^{2} d s \\
\left.\leq \alpha\left|u^{k}(0)\right|^{2}+\left|h^{k}(0)\right|^{2}\right)+2 c \int_{0}^{t}\|f(s)\|_{*}^{2} d s
\end{gathered}
$$

for $t \in[0, T]$.

Due to the choice of $u^{k}(0)$ and $h^{k}(0)$, there exists $C>0$ independent of $k$ such that $\left|u^{k}(0)\right| \leq C\left|u_{0}\right|,\left|h^{k}(0)\right| \leq C\left|h_{0}\right|$. This implies that $\left(u^{k}(t), h^{k}(t)\right)$ cannot blow up for finite $t \geq 0$, and, therefore that the maximal interval of existence is $(0, \infty)$, that is, we can take $T_{k}=+\infty$. Also, we observe that $u^{k}, h^{k}$ remains bounded in $L^{\infty}(0, T ; H) \cap L^{2}(0, T ; V)$ 
The next step in the proof consists in proving $\left(u_{f}^{k}\right)$ and $\left(h_{i}^{k}\right)$ are bounded in $L^{2}\left(0, T ; V_{n / 2}^{*}\right)$. To this end, let $u \mathrm{l}$ fix some notation; define the operatorts $A, B: V_{n / 2} \rightarrow V_{n / 2}^{*}$ hy

$$
\begin{aligned}
& \langle A u, v\rangle=a(u, v), \\
& \langle B u, v\rangle=b(u, u, v),
\end{aligned}
$$

for all $u_{1} v \in V_{n / 2}$ where $($,$) denotes the duality between V_{n / 2}$ and $V_{n / 2}$. As in the case of the classical Navier-Stokes equations, the fact that we are taking $s=n / 2$ is enough to guarante that $A$ and $B$ are well defined operators.

We will prove that $\left\{A u^{k}(t)\right\}_{k=1}^{\infty},\left\{B u^{k}(t)\right\}_{k=1}^{\infty}$ and $\left\{B h^{k}(t)\right\}_{k=1}^{\infty}$ are uniformly bounded sequences in $L^{2}\left(0, T, V_{n / 2}^{*}\right)$

Indeed, for all $v \in V_{s}$, we have

$$
\left|\left\langle A u^{k}(t), v\right\rangle\right|=\left|a\left(u^{k}(t), v\right)\right| \leq C|| u^{k}(t)\||| v\|_{s}
$$

whence, we have

$$
\left\|A u^{k}(t)\right\| v_{n / 2}=\sup _{\|v\|_{v} \neq 0} \frac{\left|\left\langle A u^{k}(t), v\right\rangle\right|}{\|v\|_{\imath}} \leq\left\|u^{k}(t)\right\|,
$$

conseçuently

$$
\int_{0}^{T}\left\|A u^{k}(t)\right\|_{V_{n / 2}^{2}}^{2} d t \leq C \int_{0}^{T}\left\|u^{k}(t)\right\|^{2} d t
$$

To prove the boundedness of $\left\{B u^{k}(t)\right\}_{k=1}^{\infty}$ in the space $L^{2}\left(0, T^{\prime}, V_{n / 2}^{*}\right)$ we will use the following interpolation result whose proof cun be found in Lions $[8]$, p. 73 .

Lemma. If $\left\{u^{k}\right\}_{k=1}^{\infty}$ is a bounded sequence in $L^{2}(0, T ; V) \cap L^{\infty}(0, T ; H)$, then $\left\{u^{k}\right\}_{k=1}^{\infty}$ is also bounded in $L^{4}\left(0, T_{;} L^{p}(\Omega)\right)$, where $\frac{1}{p}=\frac{1}{2}-\frac{1}{2 n}$.

Ueing this Lemma, conclude that

$$
\begin{aligned}
\left|\left(B u^{k}(t), v\right\rangle\right| & \leq \sum_{i, j=1}^{n}\left|u_{i}^{k}(t)\right|_{L},\left|u_{j}^{k}(t)\right|_{L,}\left|\frac{\partial v_{j}}{\partial x_{i}}\right|_{L^{n}} \\
& \leq\left. C \sum_{i, j=1}^{n}\left|u_{i}^{k}(t)\right|_{L,}\left|u_{j}^{k}(t)\right|_{L, p}|| \frac{\partial v_{j}}{\partial x_{i}}\right|_{n / 2-1} \\
& \leq C /\left.\left.u^{k}(t)\right|_{L,} ^{2}|| v\right|_{n / 2}
\end{aligned}
$$

since $\frac{1}{p}=\frac{1}{2}-\frac{1}{2 n}=1$ and the Sobolev embedding $H^{n / 2-1}(\Omega) \subseteq L^{n}(\Omega)$. This implies

$$
\int_{0}^{T}\left\|B u^{k}(t)\right\|_{V_{n / 3}^{2}}^{2} d t \leq C \int_{0}^{T}\left|u^{k}\right|_{L_{p}^{*}}^{4} d t .
$$


Thus, $\left\{B u^{k}(t)\right\}_{k=1}^{\infty}$ is uniformly bounded in $L^{2}\left(0, T ; V_{n / 2}^{*}\right)$. $L^{2}\left(0, T ; V_{n / 2}^{*}\right)$.

Similarly, we prove that $\left\{B h^{k}(t)\right\}_{k=1}^{\infty}$ is uniformly bounded in

Now, we consider the projections $P_{k}: H \rightarrow V^{k}$ defined by

$$
P_{k} u=\sum_{i=1}^{k}\left(u, w^{i}\right) w^{i}
$$

Since $V_{n / 2} \subset H$ and $V^{k} \subset V_{n / 2}$, we can consider the restriction $P_{k}$ : $V_{n / 2} \rightarrow V_{n / 2}$. It is easy to see that $P_{k} \in \mathcal{L}\left(V_{n / 2}, V_{n / 2}\right)$, hence $P_{k}^{*}: V_{n / 2}^{*} \rightarrow V_{n / 2}^{*}$ defined by $\left.\left\langle P_{k}^{*}(v), w\right\rangle=\left\langle v, P_{k} w\right)\right\rangle$ lies $\mathcal{L}\left(V_{n / 2}^{*}, V_{n / 2}^{*}\right)$ and $\left\|P_{k}^{*}\right\| \leq\left\|P_{k}\right\| \leq 1$. We also observe that the functions $w^{i}(x)$ are invariant by $P_{k}$, i.e.,

$$
P_{k}\left(w^{i}\right)=w^{i} .
$$

From the definition of $A$ and $B$ and (2.1), we conclude that

$$
\begin{aligned}
\alpha\left(u_{t}^{k}(t), w^{i}\right) & =\left\langle(-\nu A-\alpha B) u^{k}(t)+\alpha f(t)+B h^{k}(t), w^{i}\right\rangle \\
& =\left\langle P_{k}^{*}\left((-\nu A-\alpha B) u^{k}(t)+\alpha f(t)+B h^{k}(t)\right), w^{i}\right\rangle
\end{aligned}
$$

Whence, for all $w \in V^{k}$, we have

$$
\alpha\left(u_{t}^{k}(t), w\right)=\left\langle P_{k}^{*}\left((-\nu A-\alpha B) u^{k}(t)+\alpha f(t)+B h^{k}(t)\right), w\right\rangle .
$$

Therefore, by taking $w=P_{k} v$, with $v \in V_{n / 2}$, we obtain

$$
\alpha\left(u_{t}^{k}(t), v\right)=\left\langle P_{k}^{*}\left((-\nu A-\alpha B) u^{k}(t)+\alpha f(t)+B h^{k}(t), v\right\rangle,\right.
$$

and therefore

$$
u_{t}^{k}(t)=\frac{1}{\alpha} P_{k}^{*}\left((-\nu A-\alpha B) u^{k}(t)+\alpha f(t)+B h^{k}(t)\right),
$$

and we conclude that $\left\{u_{t}^{k}\right\}_{k=1}^{\infty}$, is uniformly bounded in $L^{2}\left(0, T ; V_{n / 2}^{*}\right)$ thanks to the previous estimates and the fact that $\left\|P_{k}^{*}\right\| \leq 1$.

Working as before, we have

$$
h_{t}^{k}(t)=P_{k}^{*}\left(\left(-\gamma A h^{k}(t)+E\left(u^{k}, h^{k}\right)-E\left(h^{k}, u^{k}\right)\right),\right.
$$

where $\langle E(u, w), h\rangle=b(u, w, h)$. Thus it is sufficient to show that $\left\{E\left(u^{k}, h^{k}\right)\right\}_{k=1}^{\infty}$ and $\left\{E\left(u^{k}, h^{k}\right)\right\}_{k=1}^{\infty}$ are uniformly bounded $L^{2}\left(0, T ; V_{n / 2}^{*}\right)$ to conclude that $\left\{h_{t}^{k}\right\}_{k=1}^{\infty}$ is uniformly bounded in $L^{2}\left(0, T ; V_{n / 2}^{*}\right)$.

To do this, we observe that

$$
\left|\left\langle E\left(u^{k}, h^{k}\right), h\right\rangle\right| \leq\left.\sum_{i, j}\left|u_{j}^{k}\right|_{L_{p}}\left|h_{i}^{k}\right|_{L p}\left\|\left.\frac{\partial h}{\partial x_{i}}\right|_{L^{n}} \leq C\left|u^{k}\right|\left|h^{k}\right|_{L^{p}}\right\| h\right|_{n / 2},
$$


and therefore, $\left\|E\left(u^{k}, h^{k}\right)\right\|_{V_{n / 2}^{*}}^{2} \leq C\left|u^{k}\right|\left\|h^{k}\right\|_{L^{p}}^{2}$

This implies

$$
\int_{0}^{T} \| E\left(u^{k}(t), h^{k}(t) \|_{V_{n / 2}^{*}}^{2} d t \leq C\left(\int_{0}^{T}\left|u^{k}(t)\right|_{L_{p}}^{4} d t\right)^{1 / 2}\left(\int_{0}^{T}\left|h^{k}(t)\right|_{L^{p}}^{4} d t\right)^{1 / 2} \leq C .\right.
$$

Similarly, we prove that $\left\{E\left(h^{k}, u^{k}\right)\right\}_{k=1}^{\infty}$ is uniformly bounded in $L^{2}\left(0, T ; V_{n / 2}^{*}\right)$.

Therefore, arguing as in Lions [8, p.76] and making use of the AubinLions Lemma with $B_{0}=V, p_{0}=2, B_{1}=V_{n / 2}^{*}, p_{1}=2$ and $B=H$ (see Theorem 1.5.1 and Lemma 1.5.2 in [8, p. 58]), we can conclude that there exists $u, h \in$ $L^{2}(0, T ; V)$ and subsequences we keep denoting $\left\{u^{k}\right\}_{k=1}^{\infty}\left\{h^{k}\right\}_{k=1}^{\infty}$ as before to simplify the notation, such that as $k \rightarrow+\infty$, we have:

$$
\begin{aligned}
& u^{k} \rightarrow u \text { and } h^{k} \rightarrow h \text { weakly in } L^{2}(0, T ; V) \text { and } L^{\infty}(0, T ; H) \\
& u^{k} \rightarrow u \text { and } h^{k} \rightarrow h \text { strongly in } L^{2}(0, T ; H) ; \\
& u_{t}^{k} \rightarrow u_{t} \text { and } h_{t}^{k} \rightarrow h_{t} \text { weakly } L^{2}\left(0, T ; V_{n / 2}^{*}\right) .
\end{aligned}
$$

Once these later convergences are stablished, it is a standard procedure to take the limit as $k \rightarrow+\infty$ in (2.1), along the previous subsequences (see [8, p. 76-77]) to conclude that $(u, h)$ form a weak solution of (1.1)-(1.3).

\section{Proof of Theorem 2.2}

We first prove the result of regularity. We observe that the proof of the previous Theorem shows that in case $n=2, u_{t} \in L^{2}\left(0, T ; V^{*}\right)$ and $h_{t} \in L^{2}\left(0, T ; V^{*}\right)$, since $V_{n / 2}=V$ when $n=2$. Consequently, by applying Lemma 1.2 in Temam $[12$, p. 260], we obtain that $u$ and $h$ are almost everywhere equal to a continuous function from $[0, T]$ into $H$.

We also recall that Lemma 1.2 in Temam [12, p. 260-261] asserts that the following identities hold:

$$
\frac{d}{d t}|u(t)|^{2}=2\left(u_{t}(t), u(t)\right) \text { and } \quad \frac{d}{d t}|h(t)|^{2}=2\left(h_{t}(t), h(t)\right) .
$$

These results will be used in the following proof of uniqueness:

Consider that $\left(u_{1}, h_{1}\right)$ and $\left(u_{2}, h_{2}\right)$ are two solutions of problem (1.1), (1.2) and (1.3) with the same $f$ and $u_{0}, h_{0}$; define differences $w=u_{1}-u_{2}$ and $v=h_{1}-h_{2}$. They satisfy

$$
\begin{aligned}
& \alpha\left(w_{t}, \phi\right)+\nu a(w, \phi)=-\alpha b\left(w, u_{1}, \phi\right)-\alpha b\left(u_{2}, w, \phi\right)+b\left(v, h_{1}, \phi\right)+b\left(h_{2}, v, \phi\right) \\
& \left(v_{t}, \psi\right)+\gamma a(v, \psi)=-b\left(u_{1}, v, \psi\right)-b\left(w, h_{2}, \psi\right)+b\left(v, u_{1}, \psi\right)+b\left(h_{2}, w, \psi\right) .
\end{aligned}
$$

for any $\phi, \psi \in V$; also we have $w(0)=v(0)=0$.

By the proof of Theorem 2.1, $w_{t}$ and $v_{t}$ belong to $L^{2}\left(0, T ; V^{*}\right)$.

Consequently, by setting $\phi=w$ and $\psi=v$ and integrating in $t$, we obtain 


$$
\begin{aligned}
& \left.\frac{\alpha}{2} \mid w(t)\right)\left.\right|^{2}+\nu \int_{0}^{t} a(w, w) d s+\int_{0}^{t}\left[\alpha b\left(w, u_{1}, w\right)-b\left(v, h_{1}, w\right)-b\left(h_{2}, v, w\right)\right]=0 \\
& \left.\frac{1}{2} \mid v(t)\right)\left.\right|^{2}+\gamma \int_{0}^{t} a(v, v) d s+\int_{0}^{t}\left[+b\left(w, h_{2}, v\right)-b\left(v, u_{1}, v\right)-b\left(h_{2}, w, v\right)\right]=0 .
\end{aligned}
$$

Adding the above equalities, we have

$$
\begin{aligned}
\left.\frac{\alpha}{2} \mid w(t)\right)\left.\right|^{2} & \left.+\frac{1}{2} \mid v(t)\right)\left.\right|^{2}+\nu \int_{0}^{t}\|w\|^{2} d s+\gamma \int_{0}^{t}\|v\|^{2} d s \\
= & -\int_{0}^{t}\left[\alpha b\left(w, u_{1}, w\right)+b\left(v, h_{1}, w\right)-b\left(w, h_{2}, v\right)\right. \\
& \left.+b\left(v, u_{1}, v\right)+b\left(h_{2}, w, v\right)\right] .
\end{aligned}
$$

Now, we observe that

$$
\begin{aligned}
\int_{0}^{t} \alpha b\left(w, u_{1}, w\right) d s & \leq \int_{0}^{t} c \alpha|w|_{L^{4}}^{2}\left\|u_{1}\right\| d s \\
& \leq c \alpha^{2} \int_{0}^{t}|w|\|w\|\left\|u_{1}\right\| d s \\
& \leq \frac{\nu}{6} \int_{0}^{t}\|w\|^{2} d s+C_{\nu}(\alpha) \alpha \int_{0}^{t}|w|^{2}\left\|u_{1}\right\|^{2} d s
\end{aligned}
$$

where we used the Lemma 3.3 in Temam [12], p. 261 together with Hölder and Young inequalities.

Analogously, we can prove

$$
\int_{0}^{t} b\left(v, u_{1}, v\right) d s \leq \frac{\gamma}{6} \int_{0}^{t}\|v\|^{2} d s+C_{\gamma} \int_{0}^{t}|v|^{2}\left\|u_{1}\right\|^{2} d s
$$

$\int_{0}^{t} b\left(v, h_{1}, w\right) d s \leq \frac{\nu}{6} \int_{0}^{t}\|w\|^{2} d s+\frac{\nu}{6} \int_{0}^{t}\|v\|^{2} d s+C_{\nu, \gamma}(\alpha) \int_{0}^{t}\left(|v|^{2}+\alpha|w|^{2}\right)\left\|h_{1}\right\|^{2} d s$
$\int_{0}^{t} b\left(w, h_{2}, v\right) d s \leq \frac{\gamma}{6} \int_{0}^{t}\|w\|^{2} d s+\frac{\nu}{6} \int_{0}^{t}\|v\|^{2} d s+C_{\nu, \gamma}(\alpha) \int_{0}^{t}\left(|v|^{2}+\alpha|w|^{2}\right)\left\|h_{2}\right\|^{2} d s$

By using the above inequalities in (4.1), we get

$$
\alpha|w(t)|^{2}+|v(t)|^{2} \leq C \int_{0}^{t}\left(\alpha|w(s)|^{2}+|v(s)|^{2}\right)\left(\left\|u_{1}\right\|^{2}+\left\|h_{1}\right\|^{2}+\left\|h_{2}\right\|^{2}\right) d s,
$$

where $C$ is positive constant that only depends on $\nu, \gamma, \alpha$. 
Now, the use of Gronwall inequality implies for every $t \in[0, T]$ that

$$
\alpha|w(t)|^{2}+|v(t)|^{2} \leq\left(\alpha|w(0)|^{2}+|v(0)|^{2}\right) e^{\varphi(t)},
$$

where $\varphi(t)=C \int_{0}^{t}\left(\left\|u_{1}\right\|^{2}+\left\|h_{1}\right\|^{2}+\left\|h_{2}\right\|^{2}\right) d s<+\infty$.

This last inequality, implies that $w(t) \equiv v(t) \equiv 0$ and hence that $u_{1}=u_{2} \quad$ and $\quad h_{1}=h_{2}$. Thus the uniqueness is proved and this completes the proof of Theorem 2.2 .

\section{Proof of Theorem 2.3}

From (3.1) and by recalling that $a(v, v) \geq C_{2}|v|^{2}$ for $v \in V$, we conclude that

$$
\frac{d}{d t}\left(\alpha\left|u^{k}(t)\right|^{2}+\left|h^{k}(t)\right|^{2}\right)+C_{3}\left(\alpha\left|u^{k}(t)\right|^{2}+\left|h^{k}(t)\right|^{2}\right) \leq 2 C|| f(t)||_{*}^{2}
$$

where $C_{3}=\min \left\{C_{2} \nu / \alpha, 2 C_{2} \gamma\right\}>0$. we obtain

Multiplying the above inequality by $e^{C_{3} t}$ and integrating from 0 to $T$,

$$
e^{C_{3} T}\left(\alpha\left|u^{k}(T)\right|^{2}+\left|h^{k}(T)\right|^{2}\right) \leq \alpha\left|u_{0}\right|^{2}+\left|h_{0}\right|^{2}+2 C \int_{0}^{T} e^{C_{3} t}\|f(t)\|_{*}^{2} d t
$$

We denote by $\varphi^{k}(t)$ the vector $\left(u^{k}, h^{k}\right)$ and $\left.\left\|\varphi^{k}(t)\right\|\right|^{2}=\alpha\left|u^{k}(t)\right|^{2}+$

With this notation, the above inequality is rewritten as

$$
e^{C_{3} T}\left\|\varphi^{k}(T)\right\|^{2} \leq\left\|\varphi^{k}(0)\right\|+2 C \int_{0}^{T} e^{C_{3} t}\|f(t)\|_{*}^{2} d t .
$$

Now, let's define the mapping $L^{k}:[0, T] \rightarrow \mathbb{R}^{2 k}$ as

$$
L^{k}(t)=\left(\alpha^{1 / 2} c_{1 k}(t), \ldots, \alpha^{1 / 2} c_{k k}(t), d_{1 k}(t), \ldots, d_{k k}(t)\right),
$$

where $c_{i k}(t)$ and $d_{i k}(t), i=1, \ldots, k$, are respectively the coefficient of the expansion of $u^{k}(t)$ and $h^{k}(t)$, as defined in Section 2 .

To be used later on, we observe that,

$$
\left\|L^{k}(t)\right\|_{\mathbb{R}^{2 k}}=\left\|\varphi^{k}(t)\right\|,
$$

since we have chosen the spectral basis $\left\{w^{i}(x)\right\}_{i=1}^{\infty}$ to be orthonormal in $\left(L^{2}(\Omega)\right)^{n}$.

Now, we define a mapping $\Phi^{k}: \mathbb{R}^{2 k} \rightarrow \mathbb{R}^{2 k}$ as follows: given $L_{0} \in \mathbb{R}^{2 k}$ we define $\Phi^{k}\left(L_{0}\right)=L^{k}(T)$, where $L^{k}(t)$ corresponds to the solution of problem $(2.1),(2.2)$ with initial value corresponding to $L_{0}$. It is easy to see that $\Phi^{k}$ is continuous and we want to prove that $\Phi^{k}$ has a fixed point. For this, as a consequence 
of the fixed point theorem of Brouwer, it is enough to prove that for any $\lambda \in[0,1]$, a possible solution of the equation

$$
L_{0}^{k}(\lambda)=\lambda \Phi^{k}\left(L_{0}^{k}(\lambda)\right)
$$

is bounded independent of $\lambda$.

Since $L_{0}^{k}(0)=0$, by $(5.3)$, it is enough to prove this fact for $\lambda \in(0,1]$. In this case, $(5.3)$ is equivalent to $\Phi^{k}\left(L_{0}^{k}(\lambda)\right)=L_{0}^{k}(\lambda) / \lambda$, and therefore by definition of $\Phi^{k}$ and (5.2), inequality (5.1) implies that

$$
e^{C_{3} T}\left\|L_{0}^{k}(\lambda) / \lambda\right\|_{\mathbb{R}^{2 k}}^{2} \leq\left\|L_{0}^{k}(\lambda)\right\|_{\mathbb{R}^{2 k}}^{2}+2 C \int_{0}^{T} e^{C_{3} t}\|f(t)\|_{*}^{2} d t
$$

which implies that

$$
\left\|L_{0}^{k}(\lambda)\right\|_{R^{2 k}}^{2} \leq \frac{2 C \int_{0}^{T} e^{C_{3} t}\|f(t)\|_{*}^{2} d t}{e^{C_{3} T}-1}=M
$$

since $\lambda \in(0,1]$. This bound is independent of $\lambda \in[0,1]$ and therefore $\Phi^{k}$ has a fixed point $L_{0}^{k}(1)$ satisfying the sance bound as (5.4). This corresponds to the existence of a solution $\left(u_{p}^{k}(t), h_{p}^{k}(t)\right)$ of $(2.1)$, satisfying $u_{p}^{t}(0)=u_{p}^{k}(T)$ and $h_{p}^{k}(0)=h^{k}(T)$, that is, they are periodic approximate solutions.

Moreover, $\left.\alpha\left\|u^{k}(0)\right\|\right|^{2}+\left\|h^{k}(0)\right\|^{2}=\left\|L_{0}^{k}(1)\right\|_{\mid R^{2 k}}^{2} \leq M$, which is also independent of $k$. Thus, the arguments in the proof of Theorem 2.1 can be repeated for the approximate solutions $\left(u_{p}^{k}, h_{p}^{k}\right)$, and this furnishes exactly the same kind of uniform in $k$ estimates for them, and therefore the convergence of a subsequence to a solution $\left(u_{p}, h_{p}\right)$ of $(1.1),(1.2)$ satisfying $u(0)=u(T)$ and $h(0)=h(T)$.

\section{References}

[1] Adams, R. A.: Sobolev Spaces, Academic Press, N.Y, 1975.

[2] Boldrini, J. L.; Rojas-Medar, M.A.: On a system of evolution equations of magnetohydrodynamic type: on the existence, regularity and approximations of solutions, Actas $2^{\underline{Q}}$ Congreso de Matemática Capricornio, Arica, 23-28, 1992 .

[3] Boldrini, J. L.; Rojas-Medar, M.A.: On a system of evolution equations of magnetohydrodynamic type, to appear in Mat. Contemp.

[4] Chizhonkov, E. V.: On a system of equation of magnetohydrodynamic type, Soviet Math. Dokl. 30, 542-545, 1984.

[5] Fujita, H. and Kato, T.: On the Navier-Stokes initial value problem, I, Arch. Rational Mech. Anal., 16 (1964), 269-315. 
[6] Kaniel, S.; Shinbrot, M.: A reproductive property of the Navier-Stokes equations, Arch. Rational Mech. Anakl. 24, 363-369, 1967.

[7] Lassner, G.: Über ein randanfargswert problem der mangnetohydrodynamik, Arch. Rational Mech. Anal, 25, 388-405, 1967.

[8] Lions, J. L.: Quelques méthodes de résolution des problémes aux limites nonlinéares, Dunod Gauthier-Villars, Paris, 1969.

[9] Pikelner, S. B.: Grundlagen der Kosmischen elektrodynamik, Moscou, 1966.

[10] Rojas-Medar, M. A.; Boldrini, J.L.: Global strong solutions of the equations of magnetohydrodynamic type, Research Report 1993/53, IMECCUNICAMP, Brasil, 1993, submitted.

[11] Schlüter, A.: Dynamik des plasmas, I and II, Z. Naturforsch. 5a, 72-78, $1950 ; \mathbf{6 a}, 73-79,1951$.

[12] Temam, R.: Navier-Stokes Equations, North-Holland, Amsterdam, Rev. Edit., 1979.

Received: 04 May, 1994

Marko A. Rojas-Medar and José Luiz Boldrini

Departamento de Matemática Aplicada,

IMECC - UNICAMP, CP 6065

13081-970, Campinas, SP, Brazil. 